\title{
THE INFLUENCE OF SHARED VISUAL CONTEXT ON PERFORMANCE WITH NOVEL CODES IN A REFERENTIAL COMMUNICATION GAME
}

\author{
THOMAS F. MÜLLER ${ }^{* 1}$, JAMES WINTERS ${ }^{1}$, TIFFANY MORISSEAU ${ }^{2}$, and \\ OLIVIER MORIN $^{1}$ \\ *Corresponding Author: tmueller@shh.mpg.de \\ ${ }^{1}$ Minds and Traditions Research Group, Max Planck Institute for the Science of Human \\ History, Jena, Germany \\ ${ }^{2}$ Central European University, Budapest, Hungary
}

It has been proposed that ostensive communication enables complex languages to evolve (Scott-Phillips, 2014). Successful ostensive communication, both verbal and non-verbal, must rely on a body of shared information that has been described as "common ground" (Clark, 1996). By virtue of knowing similar things, communicators are better able to infer each other's intentions. As a subset of the general phenomenon of common ground, shared visual context refers to the situation of two interlocutors building on visibly common information. The importance of shared visual context for referential communication has been extensively studied (e.g. Clark, Schreuder, \& Buttrick, 1983; Hanna, Tanenhaus, \& Trueswell, 2003), but its impact on language evolution remains an open question. Other studies (Winters, Kirby, \& Smith, 2015; Tinits, Nölle, \& Hartmann, 2017) have investigated the effects of different types of context on evolving languages, but not considered the shared aspect of this context. How important is shared visual context in the genesis of new languages through communication?

We conducted two laboratory experiments to answer the question of whether shared visual context between two interlocutors could help solving the task of establishing novel codes, and using them for communication. In both experiments, dyads of participants played a game in which one subject was tasked to choose the target colour out of an array of four possible answers, while the second subject tried to communicate the target, using black-and-white abstract symbols only. The symbols represented abstract shapes and objects that are not limited to one natural colour - e.g., a diamond, a piece of candy, a butterfly. Shared visual context was manipulated between dyads by minimizing or maximizing what portion of the array seen by the receiver was also seen by the sender: In the absent visual context condition, the senders only knew about the target colour, whereas they had full access to the three distractors in the shared visual context condition. Crucially, participants never received feedback about correct or wrong answers during the game, but could only tell their partner whether they understood the message instead. This, together with the non- 
conventionality of the symbols, ensured they were not relying on mere memorization, but actively creating novel codes during communication.

Our predictions for both experiments were preregistered on the Open Science Framework. In experiment 1, we tested 26 pairs of participants using the basic paradigm outlined above. In experiment 2 , we aimed to replicate the main results in a new sample of 48 participant pairs. Additionally, the procedure was adjusted to test whether codes established with shared visual context were more generalisable and would still be used if contexts changed. We did this by splitting the basic experiment in two halves, during which the reference space was limited to one half of the total colour space. Further changes were a refined symbol set and a perceptually controlled colour space.

The results of both experiments show, as predicted, that performance was better in pairs with shared visual context than in pairs with absent visual context (see Figure 1). This could be confirmed in mixed-effects models with maximal random effects structure. Furthermore, the models demonstrate that pairs make significant progress in accuracy over time in both experiments.
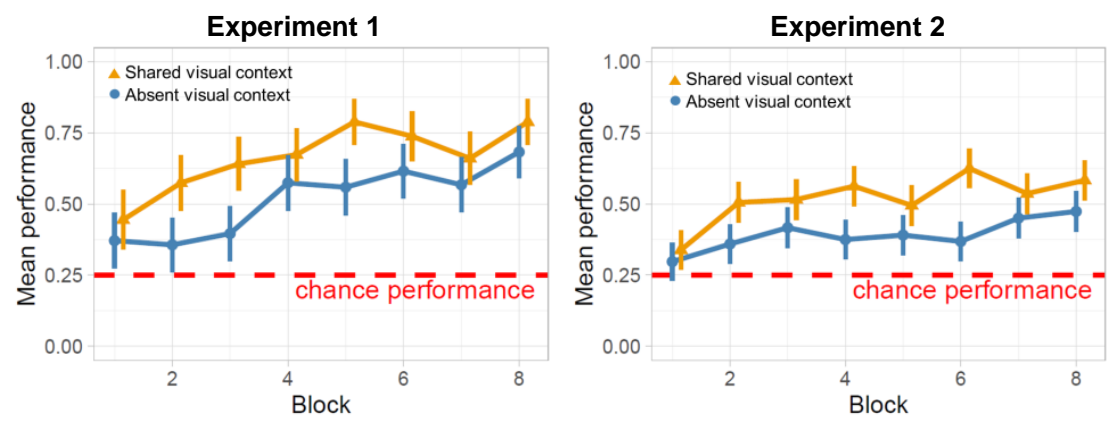

Figure 1. Performance results in both experiments. $n=26$ and 48, respectively.

Additionally, we compared the symbol inventories in both conditions in experiment 1, as measured by the number of symbols used successfully according to a preregistered threshold of successful trials, and found that symbol inventories were larger in shared visual context pairs. Lastly, we compared the generalisability of the codes in experiment 2 , measured by calculating the relative number of symbols reused in the different context of the second half of the experiment. We found that reuse was only functional with shared visual context, yet absent visual context pairs reused more symbols.

Participants successfully solved the task by establishing new codes, which vary wildly between dyads. From the results, we conclude that shared visual context has an important role during the emergence of novel codes, making them more successful and frequent. This contributes to our understanding of how communication can arise ostensively. 


\section{References}

Clark, H. H. (1996). Using language. New York, US: Cambridge University Press.

Clark, H. H., Schreuder, R., \& Buttrick, S. (1983). Common ground and the understanding of demonstrative reference. Journal of Verbal Learning \& Verbal Behavior, 22(2), 245-258.

Hanna, J. E., Tanenhaus, M. K., \& Trueswell, J. C. (2003). The effects of common ground and perspective on domains of referential interpretation. Journal of Memory and Language, 49(1), 43-61.

Scott-Phillips, T. C. (2014). Speaking our minds: Why human communication is different, and how language evolved to make it special. Palgrave MacMillan.

Tinits, P., Nölle, J., \& Hartmann, S. (2017). Usage context influences the evolution of overspecification in iterated learning. Journal of Language Evolution, 2(2), 148-159.

Winters, J., Kirby, S., \& Smith, K. (2015). Languages adapt to their contextual niche. Language and Cognition, 7(3), 415-449. 